\section{Sonar Measurement of Fetal Crown- Rump Length as Means of Assessing Maturity in First Trimester of Pregnancy}

\author{
HUGH P. ROBINSON
}

British Medical fournal, 1973 4, 28-31

\begin{abstract}
Summary
A method is reported by which the "in utero" crownrump length of the fetus may be determined by sonar in the first trimester of pregnancy. The accuracy of the technique was assessed by comparing the sonar and the direct postabortum measurements of fetuses in cases of missed abortion. A normal curve of fetal crown-rump length was derived from 214 examinations on 80 patients and by using these values in a further "blind" series it was found possible to predict the maturity of pregnancy to within three days, between the sixth and the 14th weeks of pregnancy.
\end{abstract}

\section{Introduction}

In view of the current trend in obstetrical practice of elective induction of labour an accurate knowledge of the maturity of a pregnancy is of utmost importance. The traditional method of relying on a patient's stated menstrual dates with confirmation by abdominal or vaginal examination, while reliable in most instances, may be inaccurate-especially when the date of the last menstrual period is uncertain or the menstrual cycle irregular (Beazley and Underhill, 1970). The use of sonar in such cases has been of great value, and sonar biparietal cephalometry after the 14th week of pregnancy is now regarded as the most accurate ancillary method of assessing maturity (Beazley et al., 1971). The accuracy of this technique is limited, however, because of the relatively wide biological range of normality, which increases from plus or minus one week in the second trimester of pregnancy (Campbell and Newman, 1971) to at least plus or minus two weeks towards term (Willocks et al., 1964; Campbell, 1969). It therefore follows that the earlier a measurement is performed the more reliable should be the estimation of maturity.

Pursuing this line of argument it is logical to expect that the biological range of fetal criteria should be even smaller in the first trimester of pregnancy, and thus the potential of more accurately determining maturity should be correspondingly greater. No completely objective method has yet been reported, however, by which the maturity of pregnancy at this stage may be reliably determined. The current sonar method of assessing gestational age in the first trimester depends on a subjective impression of the size of the uterus and the appearance and size of the gestation sac, with less attention being paid to the fetal

Queen Mother's Hospital, University of Glasgow, Glasgow G3 8SH HUGH P. ROBINSON, M.R.C.o.G., M.R.C. Ultrasound Research Registrar echoes (Donald and Abdulla, 1967; Donald, 1969). Growth curves of the first two of these factors as described by Hellman et al. (1969), Jouppila (1971), and Troostwijk (1972) show a range of biological variation such that they would be of little value in the accurate prediction of gestational age. Of the structures, therefore, which are readily identifiable in early pregnancy there remained only the fetus, and it followed that if sonar was to provide reliable objective information from which maturity could be calculated, then a method of measuring the fetal echoes had to be developed. It was to this end that a technique was devised by which the maximum or crown-rump length of the fetus could be measured.

\section{Method}

The apparatus used was the Diasonograph NE 4102 (Nuclear Enterprises). All the sonar scanning in this investigation was performed using a display of 0.94 to 1 . The screen graticule is calibrated to the equivalent of one-centimetre squares, and, as this graticule was used as the rule for all measurements, there was no need to correct for the above reduction factor of $6 \%$ in the display.

The technique is based on the formation of a mental threedimensional image of the position of the fetus in utero and the subsequent alignment of the scanning frame along its longitudinal axis.

With the use of the full bladder technique as described by Donald (1963) a longitudinal scan of the uterus, gestation sac, and fetal echoes is displayed on the screen. The scanning frame is then moved laterally to the patient's right until, on further scanning, the fetal echoes are barely visible within the gestation sac. By using the horizontal and vertical controls these echoes are then moved to the exact centre of the screen, as shown in fig. 1. The result of these manouvres is that one end of the fetus is now fixed "in space" using the centre of the graticule as a reference point. The scanning frame is next moved slowly to the patient's left, further scans being taken at intervals of a half to one centimetre until the fetal echo complex has almost disappeared. In the illustration the fetus is lying at an angle of less than $90 \%$ to the scanning plane with the left end of the fetus more cephalad than the right. When the serial scans (1 to 4) are made, the echoes derived from the fetus therefore appear on the screen at increasing distances from the centre of the graticule. In sonograms of longitudinal scans the left side of the picture is cephalad. If the distance moved by the fetal echoes along the horizontal or $x$-axis of the screen is "a," and the distance moved laterally by the scanning frame across the maternal abdomen is " $b$," then the angle of skew (A.S.) can be calculated by the formula tan. A.S. = a. In the clinical situation, however, it is sufficient to mark the corresponding points of each end of the fetus on the maternal abdomen and to rotate the scanning frame such that a scan may be made along a line joining the two points. Alternatively, the fetus may be positioned at an angle closer to the longitudinal than the transverse. In this situation the procedure is reversed, the angle of skew being first determined by serial transverse scans and the maximum length of the fetus determined from a longitutinal scan. A vertical component to the lie or position of the fetus is unimportant as long as the relation of the longitudinal axis of the fetus to the longitudinal and transverse axes of the scanning frame has been accurately determined. Having positioned the scanning frame as described, several scans are made a few millimetres apart until the fetal echo complex is shown at its maximum length. A Polaroid 

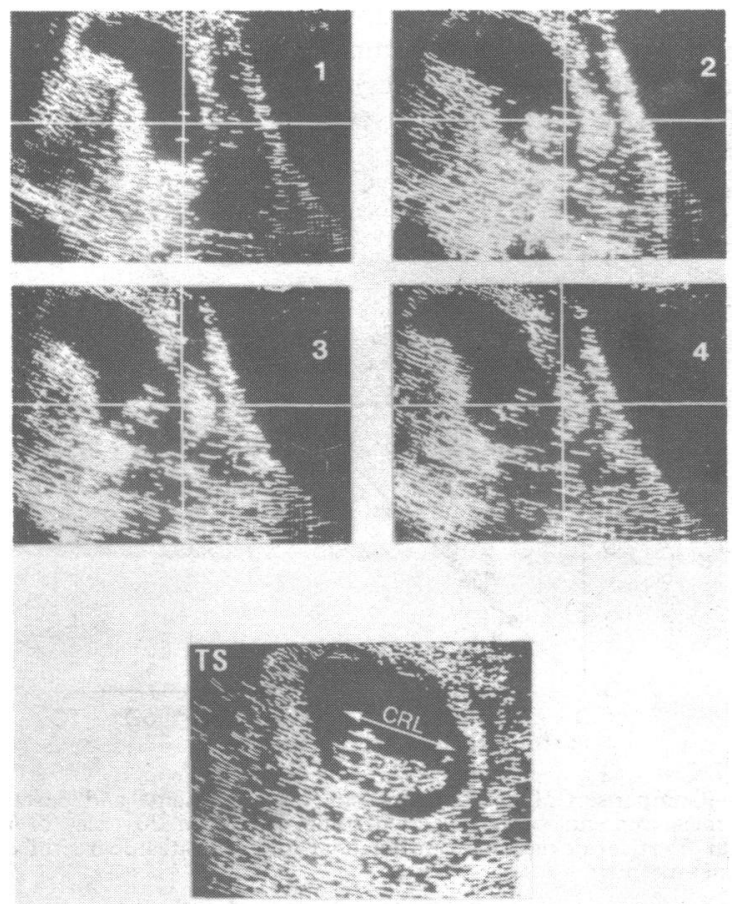

R

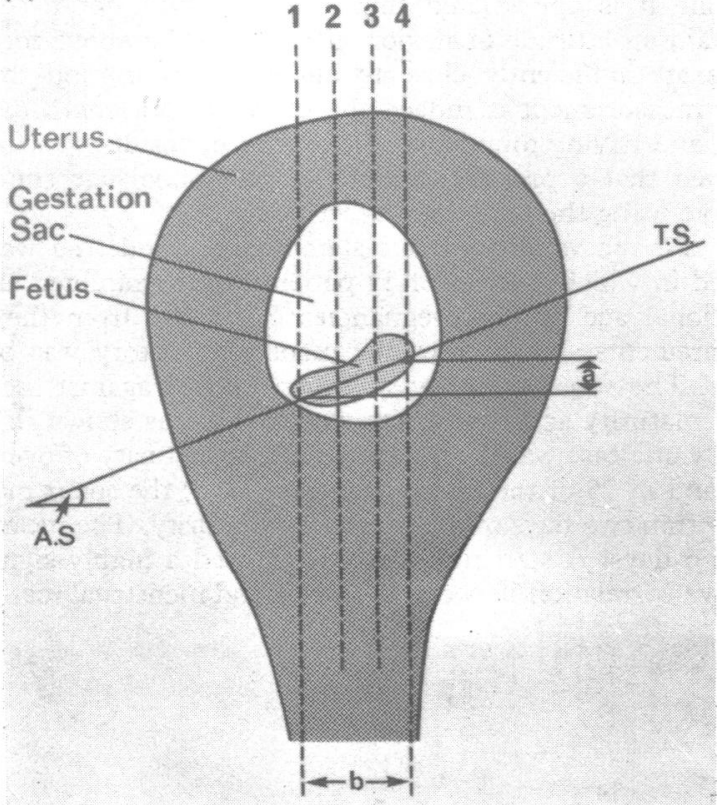

FIG. 1-Semi-diagrammatic illustration of technique involved in determining lie of fetus in utero. Vertical lines 1-4 through uterus on lower part of figure represent longitudinal scans, and distance travelled transversely by scannin frame is "b." Equivalent sonar pictures are shown above. Difference in leve between right and left ends of fetus, " $a$," is equal to distance moved by fetal echoes along $x$-axis of graticule at last longitudinal scan. Angle of longitudinal axis of fetus to horizontal (the "angle of skew" (A.S.)) may be derived from formula tan. A.S. = a (Alternatively, position of each end of fetus may be marked on patient's abdomen and a transverse scan made along line joining these two points.) Transverse scan (T.S.) has been taken at this angle of skew, along crown-rump length (C.R.L.) of fetus.

photograph is taken and the echo complex measured using the graticule as the rule. This measurement is taken to be the crown-rump length of the fetus.

At each examination fetal life is confirmed by the presence of fetal heart movement to exclude the possibility of an early missed abortion (Robinson, 1972). The rapidly increasing length of the fetus as pregnancy progresses is shown in figs. 2,3 , and 4.

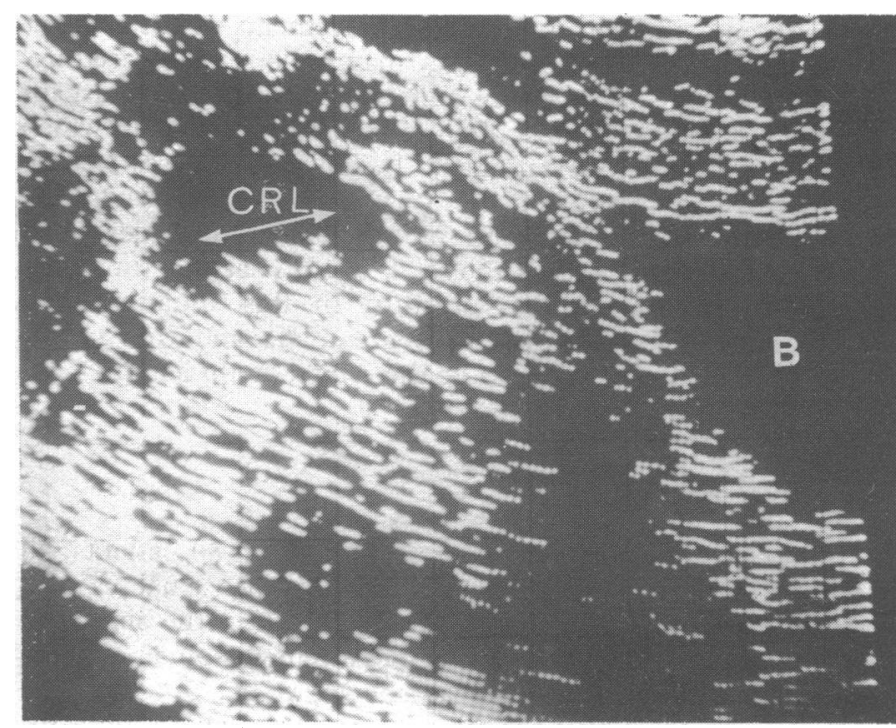

FIG. 2-Pregnancy of seven weeks and five days. Crown-rump length of fetus is $14 \mathrm{~mm}$. Scale $1: 1$. B= Bladder.

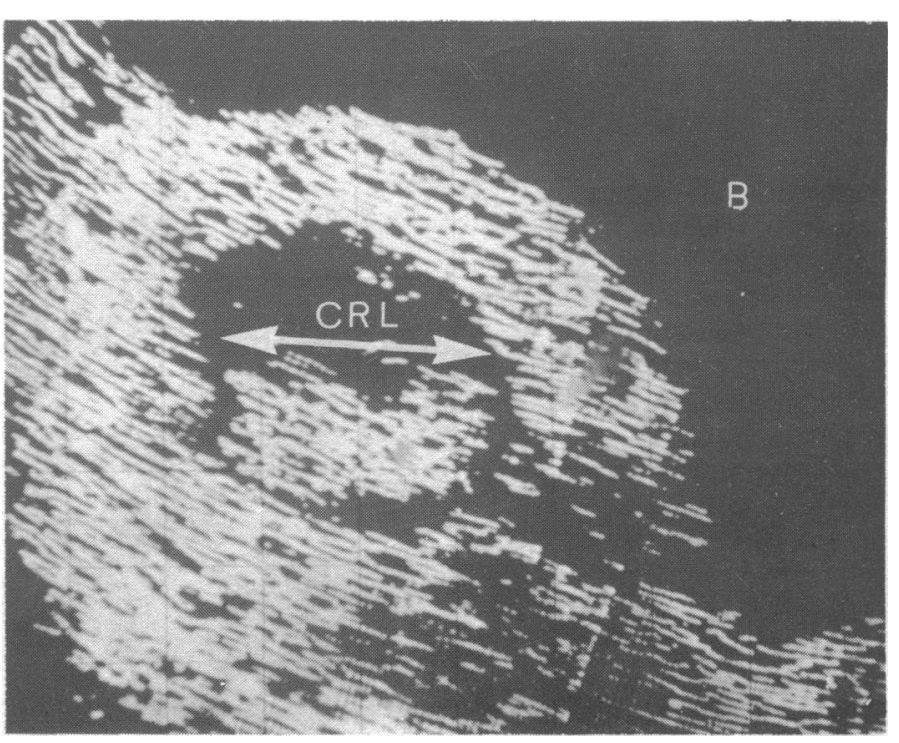

FIG. 3-Pregnancy of nine weeks and four days in which fetus has a crownrump length of $28 \mathrm{~mm}$.

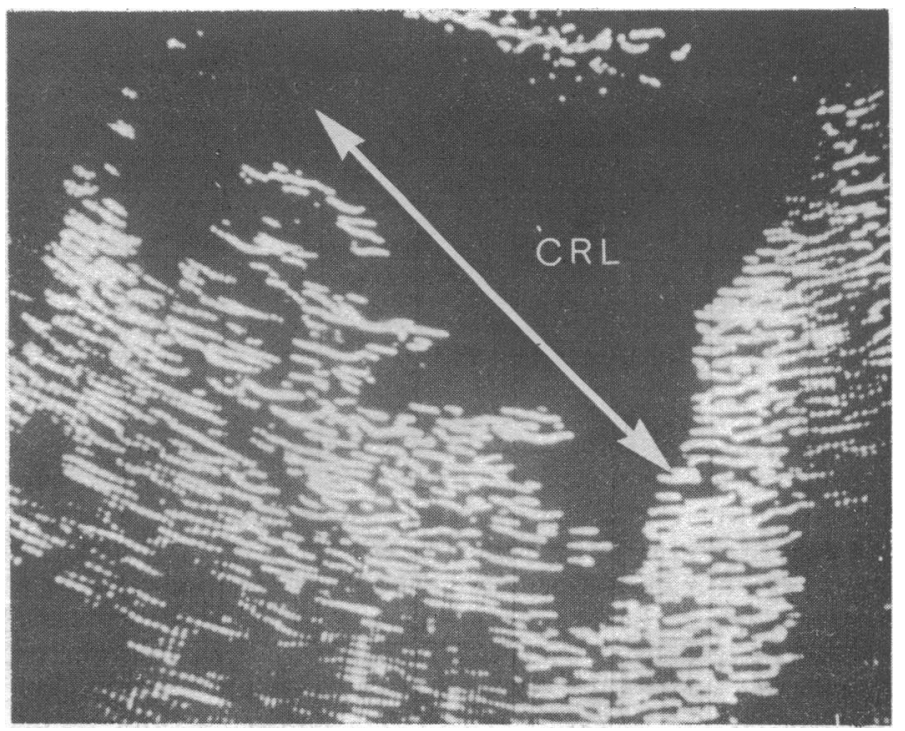

FIG. 4-Pregnancy of 12 weeks and three days with a fetal crown-rump length of $58 \mathrm{~mm}$. Head of fetus is uppermost. 
With experience only a few minutes are required to make such measurements, though random fetal movements which begin at about the ninth week of pregnancy may prolong the procedure. These movements at times are quite violent but usually last for only a few minutes and are often followed by periods in which the fetus is completely stationary. In only a few cases was no measurement possible. At the very early stages, when the crown-rump length is below $12 \mathrm{~mm}$, the technique as described is not entirely applicable as the fetal length is at this time only marginally greater than its breadth. In this situation both longitudinal and transverse scans are made and the greater length of fetal echoes taken to be the crown-rump length.

\section{Results}

A total of 214 examinations were performed on 80 patients, all of whom were certain of the dates of their last menstrual periods, had normal menstrual cycles, and were between the sixth and the 14th weeks of pregnancy. The measurements obtained were plotted against menstrual age as shown in fig. 5. From the graph it can be seen that the fetal crown rump-length increases from a mean of $10 \mathrm{~mm}$ at seven weeks to $70 \mathrm{~mm}$ at 13 weeks at a rate which increases as pregnancy advances, and that the scatter of results increases from $5 \mathrm{~mm}$ at $6 \frac{1}{2}$ weeks to $18 \mathrm{~mm}$ at $12 \frac{1}{2}$ weeks. Because of the shape of the curve, however, the absolute range of gestational age at any given crown-rump length measurement is never much more than plus or minus four days.

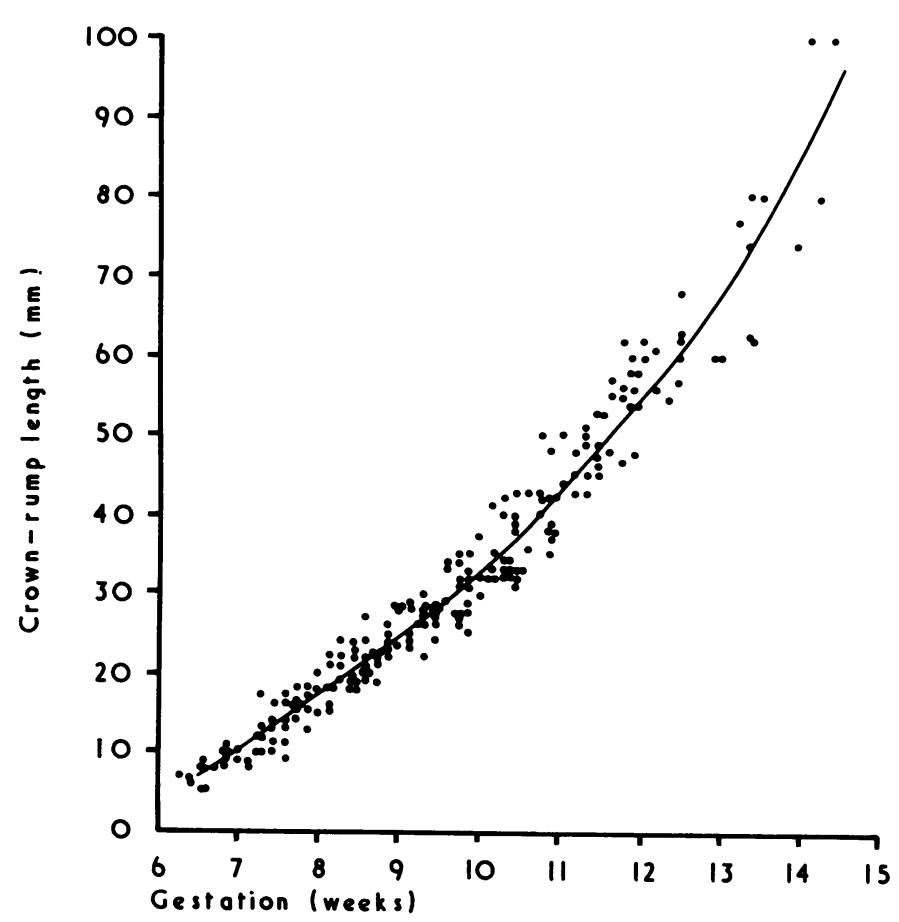

FIG. 5-Mean values and scatter of results of 214 measurements of fetal crown-rump lengths from six to 14 weeks of menstrual age.

In order to determine the accuracy of the technique "in-vivo" measurements were made of the fetuses in 20 cases of missed abortion, where death of the fetus had been confirmed by the absence of fetal heart movements. When the fetus was eventually aborted a direct measurement of the crown-rump length was made with the fetus in what was thought to be a normal degree of flexion. At the time of this measurement the corresponding sonar value was not known to the observer. The in vivo and direct measurements were compared and the results plotted as shown in fig. 6. A statistical analysis of the results showed a highly significant correlation between the values. In 16 of the 20 cases $(80 \%)$ the difference between the sonar and direct measurements was $2 \mathrm{~mm}$ or less, and the maximum difference was $4 \mathrm{~mm}$ (two cases). The mean error in this series was $1.3 \mathrm{~mm}$.

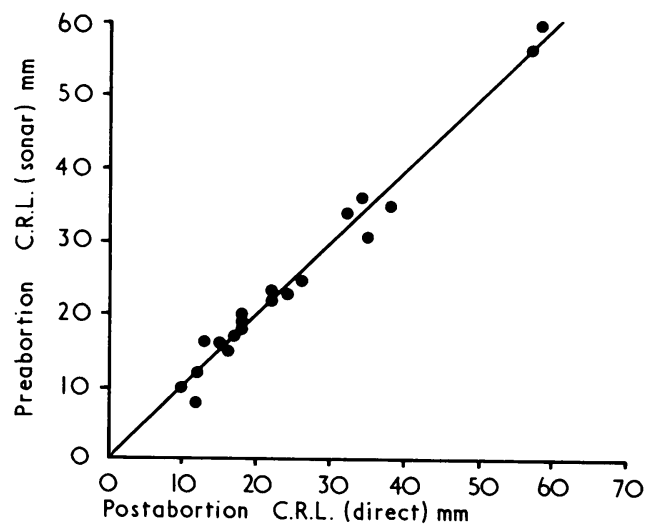
FIG. 6-Comparison of preabortion sonar measurements and postabortion abortion. Vertical deviation from diagonal line indicates degree of error of sonar measurement.

While it is appreciated that a fetus might not necessarily maintain an attitude of flexion after death the above measurements are sufficiently close to allow an assumption that the sonar measurement is indeed the crown-rump length. On this basis, and having found that the scatter of results was small, it followed that a precise estimate of gestational age should be possible using the technique described.

To test the validity of this statement a blind trial was performed in which a series of 34 patients were examined and the gestational age of the pregnancies calculated from the sonar measurements. In all cases the menstrual history was beyond doubt. The sonar maturity was then plotted against the menstrual maturity and the results were plotted as shown in fig. 7 . In only one case was there a difference in maturity of over three days and in 25 of the 34 pregnancies $(74 \%)$ the sonar maturity was within two days of the menstrual maturity. The mean error was 1.6 days. A statistical analysis showed a highly significant positive correlation between the sonar and menstrual maturities.

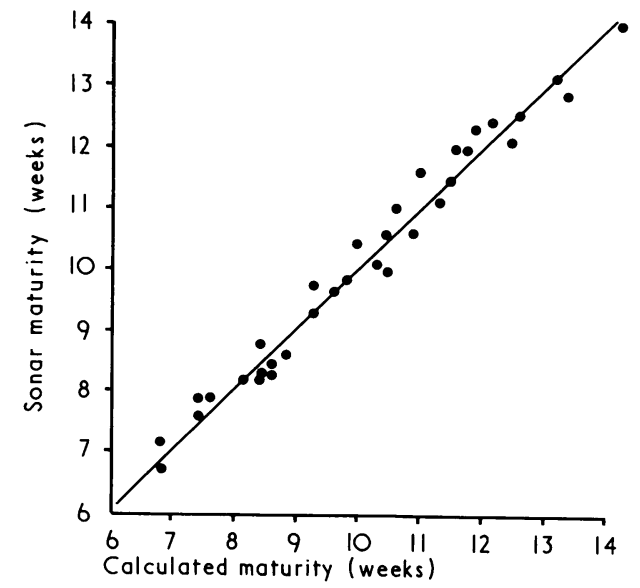

FIG. 7-Comparison of sonar and calculated menstrual maturity in 34 patients examined in a blind trial. Vertical deviation from diagonal line indicates degree of error of sonar measurement. 


\section{Discussion}

The factors contributing to the scatter of results as shown in fig. 5 are threefold. Firstly, there is a normal variation in the timing of ovulation of the order of plus or minus two days (Bell and Loraine, 1965) even in patients with normal 28-day menstrual cycles. It therefore follows that the time interval in which conception may take place will be at least four days and possibly even five days when the survival time of the ovum in the female genital tract after ovulation is considered. Secondly, it has been shown in this investigation that the inaccuracy in sonar measurement of the fetal crown-rump length is plus or minus $2 \mathrm{~mm}$. At the 10 th week this is equivalent to plus or minus one day. Finally, the range of biological variation, which, logically, would be very small in the first few weeks of gestation, would increase as pregnancy advances. This latter factor may account for the increasing scatter of results as shown in fig. 5 towards the end of the period under study.

No attempt has been made in this investigation to correlate sonar maturity with the ultimate date of delivery as it was thought that the time of spontaneous onset of labour was much more variable than the potential error involved in accepting the date of the last menstrual period when the patient is intelligent and well motivated.

Notwithstanding these factors this investigation has shown that it is possible to predict the maturity of a pregnancy between six and 14 weeks, to within three days. No other procedure allows such a degree of accuracy and reliability at this or any other stage of pregnancy, and it is now becoming our practice whenever possible to estimate in this way the maturity of "at risk" pregnancies and those where the menstrual history is in doubt.

I wish to thank Professor Ian Donald for his advice and encouragement, and the members of staff of the Queen Mother's Hospital, Glasgow, whose patients were included in this study, and Mr. J. Devlin and his department for preparing the illustrations.

This work has been undertaken with financial help from the Medical Research Council granted to Professor Ian Donald as Programme Director of a current long-term research schedule in the University of Glasgow.

\section{References}

Beazley, J. M., and Underhill, R. A. (1970). British Medical fournal, 4, 404. Bell, E. T., and Loraine, J. A. (1965), Lancet, 1, 1029.

Campbell,'S. (1969). Fournal of Obstetrics and Gynaecology of the British Commonwealth, 76, 603 .

Campbell, S., and Newman, G. B. (1971). Fournal of Obstetrics and Gynaecology of the British Commonwealth, 78, 513

Donald, I. (1963). British Medical Fournal, 2, 1154.

Donald, I., and Abdulla, U. (1967). British fournal of Radiology, 40, 604.

Donald, I. (1969). Fournal of Pediatrics, 75, 326.

Hellman, L. M., Kobayashi, M., Fillisti, L., and Lavenhar, M. (1969). American Yournal of Obstetrics and Gynecology, 103, 789 .

Jouppila, P. (1971). Acta Obstetricia et Gynecologica Scandinavica, 50, Suppl. 15.

Robinson, H. P. (1972). British Medical fournal, 4, 466.

Troostwiik, A. L. (1972). M.D. Thesis. Free University of Amsterdam.

Underhill, R. A., Beazley, J. M., and Campbell, S. (1971). British Medical fournal, 3, 736 .

Willocks, J., Donald, I., Duggan, T. C., and Day, N. (1964). fournal of Obstetrics and Gynaecology of the British Commonwealth, 71, 11.

\section{MEDICAL MEMORANDA}

\section{Meckel's Diverticulum Presenting as Fistula-in-Ano}

\author{
IAIN MCKAY， JOHN CALDER
}

British . Medical fournal, 1973, 4, 31-32

Meckel's diverticulum is a relatively common congenital abnormality, occurring in about $2 \%$ of the population. The majority of such diverticula do not cause symptoms, being found incidentally at laparotomy or necropsy. They may, however, present as acute abdominal emergencies closely mimicking acute appendicitis. Various authors have recorded rare presentations and complications of Meckel's diverticulum-for example, with peptic ulcer (Cobb, 1936), with tuberculosis (MacDonald, 1947), as perforation (Ward-McQuaid, 1950), and even a knot in Meckel's diverticulum giving rise to small-bowel obstruction (Walsh, 1950).

The following case of Meckel's diverticulum presented as fistula-in-ano. This complication has not been recorded previously.

Royal Infirmary, Glasgow G4 OSF

IAIN MCKAY, M.B., F.R.C.S., Surgical Registrar

JOHN CALDER, M.B., D.OBST.R.C.o.G., Registrar in Radiology

\section{Case Report}

A 24-year-old man was referred to the casualty department of Glasgow Royal Infirmary on 14 April 1971 as a case of "ischiorectal abscess." He gave a history of increasing pain in his left buttock of some two months duration, much worse in the two weeks preceding admission. On examination he had what appeared to be a perianal abscess on the left side. In the theatre that evening the abscess was drained of a small quantity of pus and the cavity was packed. Two days later he developed a frankly faecal discharge from the wound. Digital rectal examination yielded nothing abnormal and proctoscopy showed no internal opening of a fistula. Examination under anaesthesia was carried out and biopsy material was obtained from the rectal mucosa and wall of the perianal abscess. Both specimens were reported as showing nonspecific inflammatory changes.

On 22 April barium-meal examination showed a possible fistula between small bowel and rectum, or to the exterior direct, the barium reaching the pelvis within an hour of administration (fig. 1). Bariumenema examination a week later showed a reduction in calibre of the rectum, pronounced mucosal irregularity, and "spiking," with swelling of the anterior and left lateral walls of the rectum. These changes could have been primary or secondary to a local abscess.

The patient continued to pass flatus and faecal material through the perineal fistula, and on 1 May sinography was carried out. This showed only a localized abscess cavity without any evidence of fistulous connexion to bowel. By the following week the local inflammation had settled considerably and though there was still some discharge from the perineal wound he was allowed home, to have further investigation as an outpatient.

Barium follow-through examination on 2 June showed an ileal loop communicating with the left pararectal abscess cavity, the appearances being suggestive of an isolated area of Crohn's disease with fistula formation. The remainder of the small bowel appeared normal (fig. 2). Slight faecal leakage continued, and on 22 September a further sino- 